\title{
Inhibitory effect of new azole derivative in the corrosion of aluminum in acidic medium: common electrochemical and electrochemical noise investigation
}

\author{
A. Ehsani ${ }^{1 *}$, H. Mohammad Shiri ${ }^{2}$, M. G. Mahjani ${ }^{3}$, R. Moshrefi $^{3}$, R. Safari $^{1}$ \\ ${ }^{1}$ Department of Chemistry, Faculty of Science, University of Qom, Alghadir Blvd., Qom 3716146611, Iran \\ ${ }^{2}$ Department of Chemistry, Payame Noor University, Iran \\ ${ }^{3}$ Faculty of Chemistry, K. N. Toosi University of Technology, Tehran, Iran
}

Received 18 June 2015, received in revised form 23 October 2015, accepted 19 November 2015

\begin{abstract}
1-(2,4-Dimethylphenyl)-5-amino- $1 H$-tetrazole (DAT) nanoparticles were synthesized, and its inhibiting action on the corrosion of Aluminum AA1005 alloy (Al) in sulfuric acid was investigated using potentiodynamic polarization and electrochemical impedance spectroscopy (EIS). Inhibition efficiency increases as inhibitor concentration increases. A good correlation was observed between the potentiodynamic studies and electrochemical impedance spectroscopy with electrochemical noise measurements. Hence, it seems that the electrochemical noise (EN) method can be applied as a complementary quantitative technique to study the corrosion behavior of inhibitors. The effect of inhibitor concentration on the interpretation of noise resistance and spectral noise impedance was evaluated. Furthermore, quantum chemical calculations were employed to provide further insight into the mechanism of inhibition by DAT. It was shown that the adsorption of inhibitors on the AA1005 surface takes place through both physical and chemical adsorption. The best fit to the experimental data was obtained using Langmuir adsorption isotherm.
\end{abstract}

Ke y words: aluminum, polarization, impedance, electrochemical noise, adsorption, nanostructure

\section{Introduction}

Low cost, light weight, high thermal and electrical conductivity make aluminum an economically and industrially remarkable and important metal. The formation of a protective film on the aluminum surface is an important feature when a fresh surface of aluminum is exposed to the atmosphere or an aqueous medium. The corrosive persistence of aluminum and its oxide layer in a wide variety of aggressive solutions has been investigated. Also, the inhibition of aluminum corrosion has been extensively studied using organic and inorganic compounds. Recently, triazoles, tetrazoles, and their derivatives have been regarded as excellent corrosion inhibitors for different metallic substrates, i.e. aluminum, [1] stainless steel [2] and mild steel [3] due to the presence of nitrogen as a functional group and p electrons in their structures. These compounds are adsorbed on the metal surfaces and form a protective film, which blocks the active sites on the surface and prevents further corrosion of the metal. Benzotriazole and aminotetrazole are the common azole-based compounds the corrosion inhibition properties of which have been investigated by various techniques $[4,5]$. Benzotriazole has been known for its great versatility from drug precursors to the application in restrainer photographic emulsions. Aminotetrazoles are important heterocyclic compounds applied in the pharmaceutical area, explosive and information recording systems as ligands and precursors to a variety of nitrogen-containing compounds [6].

The present work intends to study the corrosion behavior of $\mathrm{Al}$ samples in $1 \mathrm{M} \mathrm{H}_{2} \mathrm{SO}_{4}$ solution with DAT as the organic inhibitor by common electrochemical tools, in particularly electrochemical noise analysis method. The effect of inhibitor concentration 
(a)<smiles>Cc1ccc(-n2nnnc2N)c(C)c1</smiles>

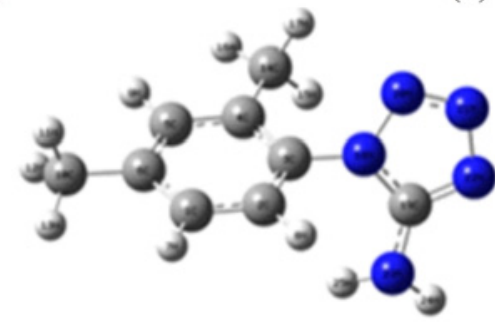

Fig. 1. (a) Structure of 1-(2,4-Dimethylphenyl)-5-amino-1H-tetrazole; (b) Optimized molecular structure of 1-(2,4-Dimethylphenyl)-5-amino- $1 H$-tetrazole.

on the interpretation of noise resistance and spectral noise impedance was evaluated. It is intended to seek the correlation between noise resistance and polarization resistance on one hand and the correlation of spectral noise impedance and electrochemical impedance spectroscopic results at a high-frequency region on the other.

\section{Experimental}

\subsection{Materials}

The employed working electrodes with surface area of $3 \mathrm{~mm}^{2}$ were prepared from aluminum AA1005 with composition of Al: 99.8, Ni: 0.6601, Si: 0.1, Mn: 0.006, $\mathrm{Mg}$ : 0.005, Cu: 0.01, Pb: 0.03, Bi: 0.005, Co: 0.002, Ti: 0.002, Na: 0.001, Fe: 0.05, and Ga: 0.005 (wt.\%). The electrolyte was $1.0 \mathrm{M} \mathrm{H}_{2} \mathrm{SO}_{4}$ solution, which was prepared using reagents obtained from Merck Chemical Co. and distilled water at ambient temperature. 1-(2,4-Dimethylphenyl)-5-amino- $1 H$-tetrazole nanostructure was prepared from a mixture of the 2,4-dimethylphenylcyanamide, $\mathrm{NaN}_{3}$, and $\mathrm{ZnCl}_{2}$ under ultrasonic irradiation. Molecular structure of the inhibitor is shown in Fig. 1.

\subsection{Testing methods}

The specimens were connected to a copper wire at one end and sealed using epoxy resin with the other end exposed as the WE surface. The surface of working electrode was abraded with silicon carbide paper (from 600 to 1200), cleaned with distilled water, and degreased using acetone before each run. A standard electrochemical cell with three electrodes in a Faraday cage was used for electrochemical noise (EN) measurements. A Faraday cage was used in order to avoid interferences with external electromagnetic field and stray currents during EN measurements. Two nominally identical electrodes (with $3 \mathrm{~mm}^{2}$ surface area) and a standard calomel electrode were used as working

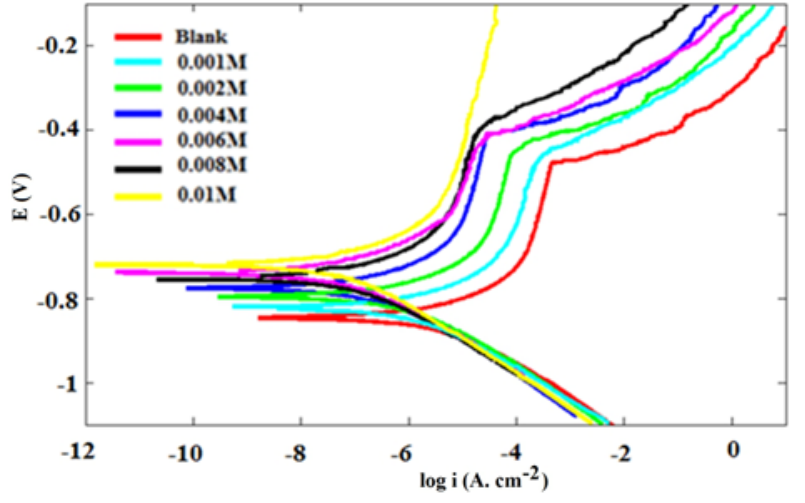

Fig. 2. Polarization curves for $\mathrm{Al}$ in $1.0 \mathrm{~mol} \mathrm{~L}^{-1} \mathrm{H}_{2} \mathrm{SO}_{4}$ in the absence and presence of different concentration of DAT at $25^{\circ} \mathrm{C}$.

and reference electrodes, respectively. The EN measurement was monitored by electrochemical interface model 1287 Solarton for $2 \mathrm{~h}$. The signal processing was carried out by Matlab 2011a software. Electrochemical potential and current noise were obtained with $0.1 \mathrm{~Hz}$ sampling frequency. For electrochemical impedance spectroscopy (EIS) and potentiodynamic polarization, a standard electrochemical cell with three electrodes including AA1005, graphite, and standard calomel electrode as working, counter, and reference electrode was used. The EIS and potentiodynamic polarization tests were performed by the potentiostat/galvanostat model 273 A EG\&G and SI (HF frequency response analyzer). The EIS measurements were carried out at an open-circuit potential with an amplitude of $10 \mathrm{mV}$ $\mathrm{AC}$ potential in the frequency range of $100 \mathrm{kHz}-100$ $\mathrm{mHz}$. The potentiodynamic current-potential curves were recorded by sweeping the electrode potential automatically from -0.5 to $0.5 \mathrm{~V}$ vs. OCP with a scanning rate of $10 \mathrm{mV} \mathrm{s}^{-1}$.

\section{Results and discussion}

\subsection{Potentiodynamic polarization}

Figure 2 shows the potentiodynamic polarization curves of AA1005 in $1.0 \mathrm{~mol} \mathrm{~L}^{-1} \mathrm{H}_{2} \mathrm{SO}_{4} \mathrm{so}^{-}$ lution in the absence and presence of various concentrations of DAT. Polarization measurements provide valuable information about the kinetics of anodic and cathodic reactions. The relevant parameters, namely corrosion current density $\left(i_{\text {corr }}\right)$, corrosion potential $\left(E_{\text {corr }}\right)$, anodic and cathodic Tafel slopes $\left(\beta_{\mathrm{a}}, \beta_{\mathrm{c}}\right)$ and polarization resistance $\left(R_{\mathrm{p}}\right)$ are listed in Table 1. According to the data presented, corrosion current density decreased as the concentration of inhibitor increased. The addition of DAT to acidic media affected anodic branches of the poten- 
Table 1. Polarization parameters and the corresponding inhibition efficiencies for $\mathrm{Al}$ in $1.0 \mathrm{~mol} \mathrm{~L}^{-1} \mathrm{H}_{2} \mathrm{SO}_{4}$ containing different concentration of DAT

\begin{tabular}{|c|c|c|c|c|c|c|}
\hline$C\left(\mathrm{~mol} \mathrm{~L}^{-1}\right)$ & $E_{\text {corr }}(\mathrm{mV})$ & $I_{\text {corr }}\left(\mu \mathrm{A} \mathrm{cm}{ }^{-2}\right)$ & $\beta_{\mathrm{c}}\left(\mathrm{mV}\right.$ decade $\left.^{-1}\right)$ & $\beta_{\mathrm{a}}\left(\mathrm{mV}\right.$ decade $\left.^{-1}\right)$ & $R_{\mathrm{p}}(\mathrm{k} \Omega)$ & $\eta$ \\
\hline 0 & -845 & 449 & 181 & 192 & 5.4 & - \\
\hline 0.001 & -820 & 364.1 & 180 & 187 & 6.6 & 18.90 \\
\hline 0.002 & -793 & 283.2 & 179 & 186 & 9.5 & 36.92 \\
\hline 0.004 & -775 & 225.5 & 182 & 179 & 11.5 & 49.77 \\
\hline 0.006 & -750 & 173.1 & 180 & 175 & 12.5 & 61.44 \\
\hline 0.008 & -736 & 136.1 & 180 & 171 & 14.6 & 69.68 \\
\hline 0.01 & -719 & 125.6 & 183 & 175 & 15.9 & 72.02 \\
\hline
\end{tabular}

tiodynamic polarization curves. Therefore, DAT behaved as an anodic inhibitor. The considerable corrosion potential shift to positive direction confirmed that the inhibitor affected the anodic reaction more in comparison to the cathodic one. The corrosion inhibition efficiency was calculated using the relation [1]:

$$
\eta(\%)=100\left(\frac{i_{\mathrm{corr}}^{*}-i_{\mathrm{corr}}}{i_{\mathrm{corr}}^{*}}\right)
$$

where $i_{\text {corr }}^{*}$ and $i_{\text {corr }}$ are uninhibited and inhibited corrosion current densities, respectively, obtained from potentiodynamic polarization curves. The $\eta$ values show that the inhibition is more pronounced with increasing inhibitor concentration. In the calculation of surface coverage, $\theta$, it was assumed that inhibition is mainly due to the blocking of active sites on the surface and hence $\theta=\eta / 100$. An attempt was made to test the Langmuir, Temkin, and Frumkin isotherms. Langmuir adsorption isotherm was found to fit well with the experimental data, as shown in Fig. 3. Langmuir isotherm is defined as:

$$
\theta=\frac{K C}{1+K C}
$$

which can be rearranged as:

$$
\frac{C}{\theta}=C+\frac{1}{K}
$$

where $\theta$ is the surface coverage, $C$ is the inhibitor concentration, and $K$ is the adsorption equilibrium constant. The plot of $C / \theta$ versus $C$ for DAT yields a straight line with a correlation coefficient close to 1.0, indicating that adsorption of this inhibitor is well described by Langmuir adsorption isotherm. In Langmuir isotherm, it is assumed that all the adsorption sites are equivalent, and the probability of the adsorption of DAT molecules at the active sites is independent of the occupation of nearby sites.

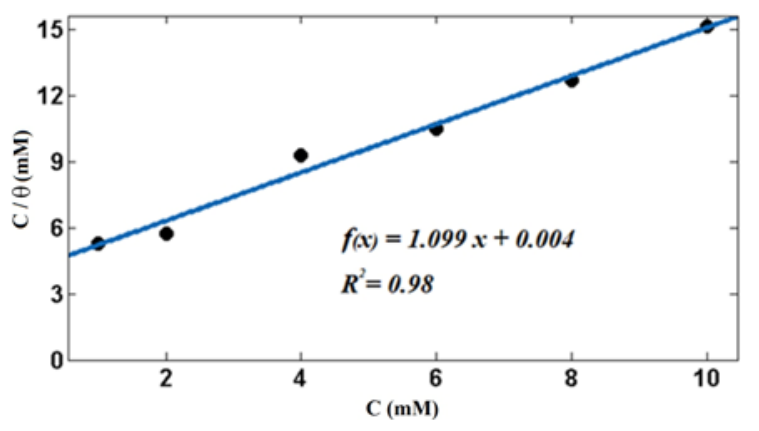

Fig. 3. Langmuir adsorption isotherm of the inhibitors by using surface coverage value calculated by Tafel polarization results.

\subsection{Electrochemical impedance spectroscopy}

Electrochemical impedance spectroscopy is an excellent technique, which has been used to understand the mechanism of corrosion, passivation phenomena, and charge transfer mechanism at the electrolyte/electrode interface. This has been broadly discussed in the literature using a variety of theoretical models [7-16]. Nyquist presentations of EIS for AA1005 in $1.0 \mathrm{~mol} \mathrm{~L}^{-1} \mathrm{H}_{2} \mathrm{SO}_{4}$ in the absence and presence of various concentrations of DAT are shown in Fig. 4. It is apparent from Fig. 4 that the impedance response of AA1005 changes significantly with increasing DAT concentration. The electrical equivalent circuit employed for the analysis of the impedance plots is shown in Fig. 4. $R_{\mathrm{s}}$ and $R_{\text {ct }}$ are the solution and charge transfer resistances, respectively. The ideal capacitive behavior is not observed in this case, and hence, a constant phase element CPE is introduced in the circuit to give a more accurate fit [1]. The impedance of the constant phase element (CPE) is defined as follows:

$$
Z_{\mathrm{CPE}}=\frac{1}{Y_{0}(j w)^{n}},
$$

where $Y_{0}$ is the $\mathrm{CPE}$ constant $\left(\mathrm{F} \mathrm{cm}^{-2} \mathrm{Sn}^{-1}\right.$ or $\left.s^{n} \Omega^{-1} \mathrm{~cm}^{-2}\right), j$ equals $\sqrt{-1}, \omega$ is the angular frequency, and $n$ is the CPE exponent. Depending on 


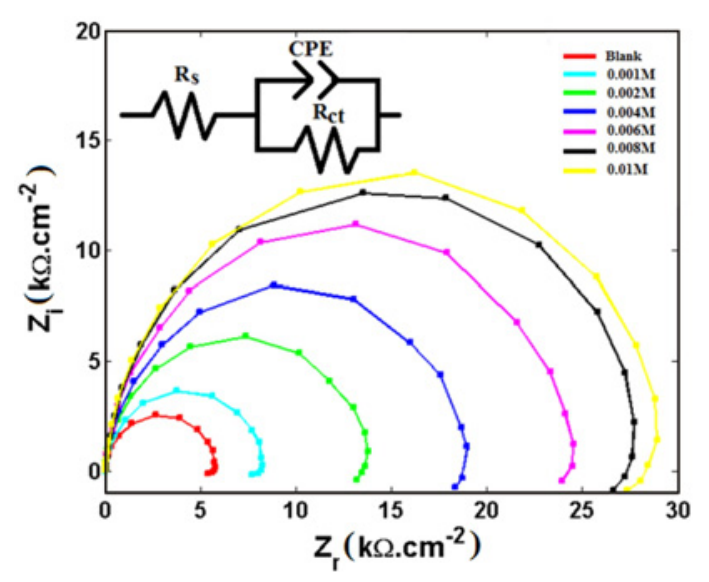

Fig. 4. Nyquist plots for $\mathrm{Al}$ in $1.0 \mathrm{~mol} \mathrm{~L}{ }^{-1} \mathrm{H}_{2} \mathrm{SO}_{4}$ in the absence and presence of different concentration of DAT at $25^{\circ} \mathrm{C}$.

$n, \mathrm{CPE}$ can represent resistance $\left(n=0, Z_{\mathrm{CPE}}=R\right)$, capacitance $\left(n=1, Z_{\mathrm{CPE}}=C\right)$, inductive $(n=1$, $\left.Z_{\mathrm{CPE}}=L\right)$ or Warburg impedance $\left(n=0.5, Z_{\mathrm{CPE}}=\right.$ $W)$. The correct equation to convert the CPE constant, $Y_{0}$, into the double layer capacitance, $C_{\mathrm{dl}}$, is the following equation:

$$
C_{\mathrm{d} 1}=Y_{0}\left(\omega_{\max }\right)^{n-1}
$$

where $\omega_{\max }$ is the angular frequency at which the imaginary component of the impedance is maximum. As observed in Table 2, the $R_{\mathrm{ct}}$ values increased as the concentration of inhibitors increased. On the other hand, the values of $C_{\mathrm{dl}}$ decreased with increasing inhibitor concentration. This was due to the increasing surface coverage by the inhibitor and consequently a decrease in local dielectric constant and/or an increase in the thickness of the double layer. This can be attributed to the replacement of water molecules with adsorbed inhibitors on the metal surface [1]:

$$
\operatorname{Org}_{\mathrm{aq}}+n \mathrm{H}_{2} \mathrm{O}_{\mathrm{ads}} \leftrightarrow \mathrm{Org}_{\mathrm{ads}}+n \mathrm{H}_{2} \mathrm{O}_{\mathrm{aq}} .
$$

Inhibition efficiencies in Table 2 were calculated through the following expression [1]:

$$
\eta_{\mathrm{EIS}}(\%)=100\left(\frac{R_{\mathrm{ct}}^{*}-R_{\mathrm{ct}}}{R_{\mathrm{ct}}^{*}}\right)
$$

where $R_{\mathrm{ct}}^{*}$ and $R_{\mathrm{ct}}$ represent charge transfer resistance before and after addition of the inhibitor to the corrosion environment, respectively. Inhibition efficiencies increased with increased inhibitor concentration. The $\eta_{\text {EIS }}$ values are in good agreement with $\eta$ obtained from the polarization method. As observed in Fig. 5, Langmuir isotherm was fitted to obtain the experimental data.

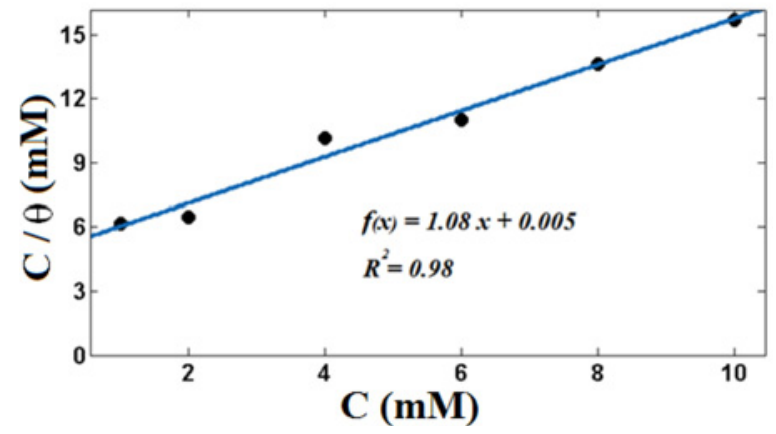

Fig. 5. Langmuir adsorption isotherm of the inhibitors by using surface coverage value calculated by electrochemical impedance spectroscopy results.

\subsection{Electrochemical noise}

The electrochemical noise is the measurement of potential and current noise with time. Potential and current are simultaneously measured without applying external perturbation. In this technique, two nominally identical electrodes are connected galvanically through a zero resistance ammeter (ZRA). The current between two electrodes is measured by ZRA. Moreover, two electrodes sense similar potentials due to short circuit connection between them, and their potential is measured toward a standard reference electrode or an identical third electrode [17]. The most popular approaches to the analysis of electrochemical noise are statistical and spectral methods [18]. The statistical method is determination and tracking of mean potential and current values, which are related to corrosion process thermodynamics and corrosion rates, respectively. The noise resistance, $R_{\mathrm{n}}$, is another important parameter, which has a physical concept like polarization resistance and is calculated by dividing standard variation of potential on the standard variation of current. In spectral methods, time domain potential and current noise are transferred to the frequency domain. The power spectral densities (PSD) are curves frequently used in the spectral analysis of electrochemical noise methods. The spectral noise impedance, $R_{\mathrm{sn}}$, is a concept similar to impedance values, which is determined by $\mathrm{AC}$ polarization techniques. This quantity has been linked to the equivalent electrochemical impedance of electrodes, which can provide much valuable information regarding the corrosion process and rate [19]. The spectral noise impedance is defined in terms of power spectral densities:

$$
R_{\mathrm{sn}}(f)=\sqrt{\frac{\operatorname{PSD}_{\mathrm{E}}(f)}{\operatorname{PSD}_{\mathrm{I}}(f)}} .
$$

However, the first and crucial step toward a comprehensive noise analysis is data pretreatment. In most electrochemical noise measurements, a DC drift ap- 
Ta b l e 2. Impedance parameters and the corresponding inhibition efficiency values for $\mathrm{Al}$ in $1.0 \mathrm{~mol} \mathrm{~L}^{-1} \mathrm{H}_{2} \mathrm{SO}_{4} \mathrm{containing}$ different concentration of DAT

\begin{tabular}{ccccccc}
\hline$C\left(\mathrm{~mol} \mathrm{~L}^{-1}\right)$ & $R_{\mathrm{s}}\left(\Omega \mathrm{cm}^{2}\right)$ & $R_{\mathrm{ct}}\left(\Omega \mathrm{cm}^{2}\right)$ & $n$ & $10^{6} Y_{0}\left(\mathrm{~F} \mathrm{~cm}^{-2} \mathrm{Sn}^{-1}\right)$ & $C_{\mathrm{dl}}\left(\mathrm{F} \mathrm{cm}^{-2}\right)$ & $\eta_{\mathrm{EIS}}(\%)$ \\
\hline 0 & 8.86 & 6450 & 0.96 & 5.03 & 4.32 & - \\
0.001 & 8.52 & 7850 & 0.96 & 3.52 & 2.02 & 17.83 \\
0.002 & 8.28 & 13687 & 0.96 & 2.67 & 1.99 & 64.67 \\
0.004 & 7.69 & 18393 & 0.96 & 2.28 & 1.95 & 73.18 \\
0.006 & 7.62 & 24050 & 0.95 & 2.25 & 1.60 & 75.56 \\
0.008 & 7.08 & 26394 & 0.95 & 1.94 & 1.07 & 78.42 \\
0.01 & 6.84 & 29894 & 0.95 & 1.27 & & \\
\hline
\end{tabular}

Table 3. Statistical analysis of electrochemical noise of $\mathrm{Al}$ in $1.0 \mathrm{~mol} \mathrm{~L}{ }^{-1} \mathrm{H}_{2} \mathrm{SO}_{4}$ containing different concentration of inhibitor (DAT)

\begin{tabular}{ccccccc}
\hline$C\left(\mathrm{~mol} \mathrm{~L}^{-1}\right)$ & $E_{\mathrm{m}}(\mathrm{V})$ & $I_{\mathrm{m}}(\mathrm{A})$ & $E_{\text {std }}$ & $I_{\mathrm{std}}$ & $R_{\mathrm{n}}(\mathrm{k} \Omega)$ & $\eta_{\mathrm{n}}(\%)$ \\
\hline 0 & -0.7469 & $4.79 \times 10^{-7}$ & $3.24 \times 10^{-3}$ & $3.129 \times 10^{-7}$ & 10.3540 & - \\
0.001 & -0.7615 & $4.79 \times 10^{-9}$ & $6.43 \times 10^{-4}$ & $4.74 \times 10^{-9}$ & 13.553 & 25 \\
0.002 & -0.7034 & $1.36 \times 10^{-7}$ & $1.68 \times 10^{-4}$ & $8.50 \times 10^{-9}$ & 19.764 & 47 \\
0.004 & -0.7124 & $4.36 \times 10^{-7}$ & $9.26 \times 10^{-5}$ & $3.65 \times 10^{-9}$ & 25.313 & 60 \\
0.006 & -0.6924 & $9.19 \times 10^{-7}$ & $5.54 \times 10^{-5}$ & $2.25 \times 10^{-9}$ & 29.055 & 65 \\
0.008 & -0.6632 & $9.93 \times 10^{-9}$ & $5.57 \times 10^{-5}$ & $1.72 \times 10^{-9}$ & 32.253 & 68 \\
0.01 & -0.6454 & $1.43 \times 10^{-7}$ & $1.89 \times 10^{-5}$ & $5.24 \times 10^{-10}$ & 36.055 & 72 \\
\hline
\end{tabular}
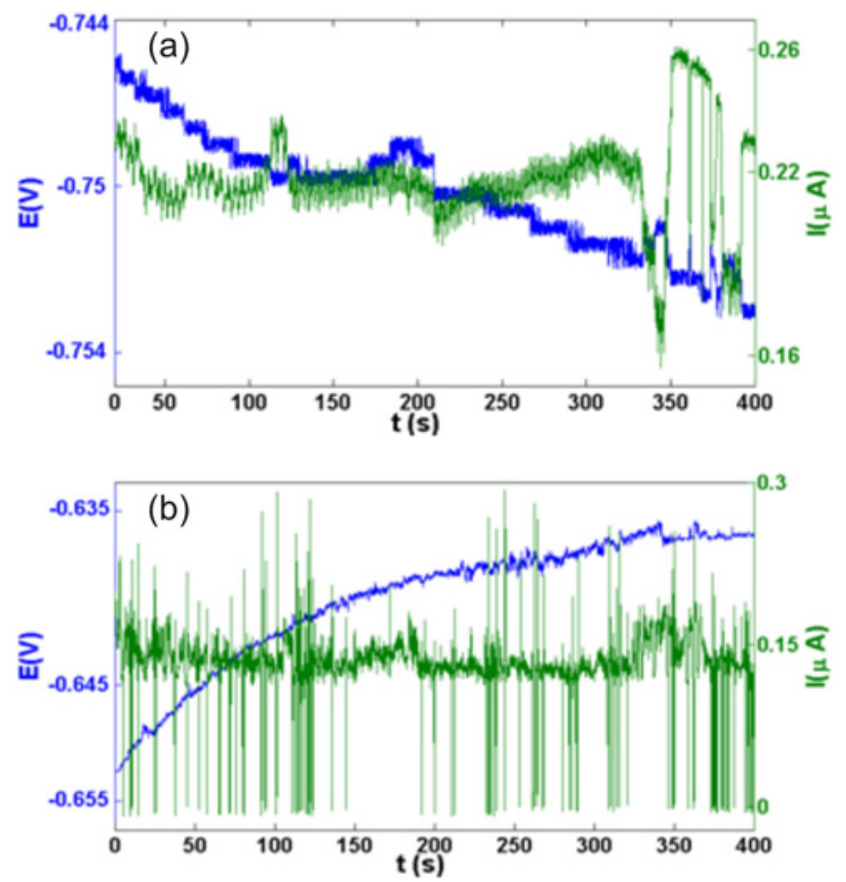

Fig. 6. Time records of electrochemical potential and current noise for $\mathrm{Al}$ in $1.0 \mathrm{~mol} \mathrm{~L}^{-1} \mathrm{H}_{2} \mathrm{SO}_{4}$ in the absence (a) and $0.001 \mathrm{~mol} \mathrm{~L}^{-1}$ (b) of DAT at $25^{\circ} \mathrm{C}$.

pears in potential or current noise data. The DC drift component can be a consequence of surface passivation for potential data or the support of one electrode for a faster anodic reaction compared to another demanding more cathodic current for current data [18]. It is believed that the DC trend should be removed before noise resistance and spectral noise impedance calculation. Moreover, to avoid the integration effect in FFT method, some extra pretreatment is needed. For this purpose, the Hann window is recommended to apply on data before transferring data from time domain to frequency domain. The obtained potential and current for $\mathrm{Al}$ electrodes in $1 \mathrm{~mol} \mathrm{~L}^{-1} \mathrm{H}_{2} \mathrm{SO}_{4}$ in the presence $(0.01 \mathrm{M})$ and absence of inhibitor DAT are shown in Fig. 6. The statistical analysis of EN data is listed in Table 3. As observed in Fig. 6, the potential and current oscillate periodically. The mean values of potential are shifted toward more positive values with increasing inhibitor concentration and numbers of birthdeath transient increase. The birth-death transients, which can be seen as a sudden increase and decrease in current values are attributed to activation controlled processes such as metastable pitting. Moreover, with increasing inhibitor concentration, the standard deviation of potential and current noise decreases, and the noise resistance value increases as a consequence. The inhibition efficiencies in Table 3 were calculated from the following expression:

$$
\eta_{\mathrm{n}}(\%)=100\left(\frac{R_{\mathrm{n}}^{*}-R_{\mathrm{n}}}{R_{\mathrm{n}}^{*}}\right),
$$

where $R_{\mathrm{n}}^{*}$ and $R_{\mathrm{n}}$ represent the charge transfer resistance before and after addition of the inhibitor to 


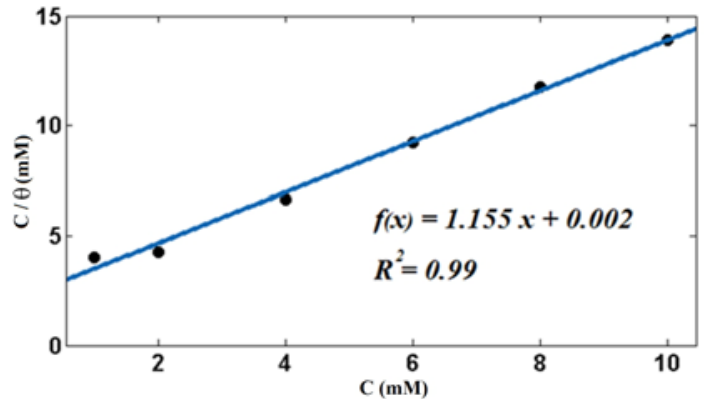

Fig. 7. Langmuir adsorption isotherm of the inhibitors by using surface coverage value calculated by electrochemical noise results.

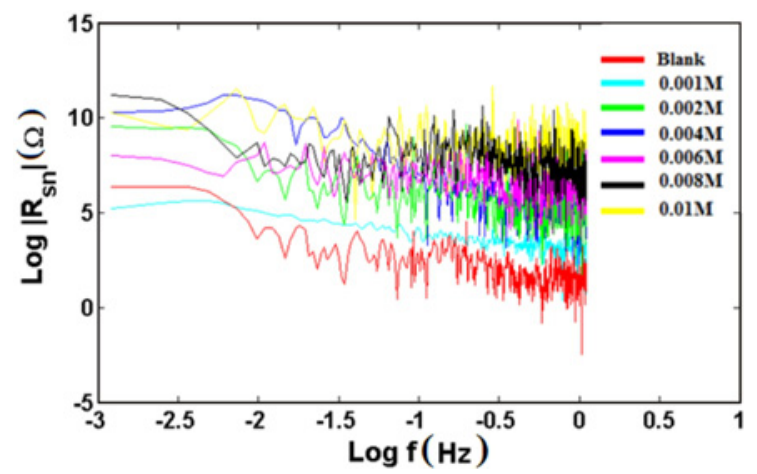

Fig. 8. Spectral noise impedance $\mathrm{Al}$ in $1.0 \mathrm{~mol} \mathrm{~L}^{-1} \mathrm{H}_{2} \mathrm{SO}_{4}$ in the absence and presence of different concentration of DAT at $25^{\circ} \mathrm{C}$.

the corrosion environment, respectively. Inhibition efficiencies increased with increasing inhibitor concentration. The $\eta_{\mathrm{n}}$ values are in good agreement with $\eta$ obtained from the polarization method and electrochemical impedance spectroscopy. Figure 7 shows the fitting of Langmuir isotherm on experimental data. The computed spectral noise impedances of electrochemical potential and current noises are shown in Fig. 8. The spectral noise impedance was computed after removing DC trend and applying a Hann window on potential and current noise data. As it can be seen, the spectral noise impedance curves moved toward higher values with increasing inhibitor concentration. However, the magnitudes are not close to those obtained from other methods due to necessary pretreatment of data, while the trends show a good correlation with obtained results from polarization methods, electrochemical impedance spectroscopy, and noise resistance.

\section{Conclusion}

The adsorption and inhibition effects of DAT nanoparticles on corrosion behavior of aluminum
AA1005 alloy in 1 mol L ${ }^{-1} \mathrm{H}_{2} \mathrm{SO}_{4}$ were studied using electrochemical techniques. Inhibition efficiency increases with increasing inhibitor concentration. A good correlation was observed between the potentiodynamic studies and electrochemical impedance spectroscopy with electrochemical noise measurements. Hence, it seems that the EN method can be applied as a complementary quantitative technique to study the corrosion behavior of inhibitors. Moreover, EIS plots indicate that the charge transfer resistance increases with increasing concentration of the inhibitor. The best fit to the experimental data was Langmuir adsorption isotherm. It was shown that the adsorption of both inhibitors on the AA1005 surface takes place through both physical and chemical adsorption.

\section{Acknowledgements}

We gratefully acknowledge the support of this work by Iranian Nano Council, Qom University and K. N. Toosi University of Technology Research Councils.

\section{References}

[1] Ehsani, A., Nasrollahzadeh, M., Mahjani, M. G., Moshrefi, R., Mostaanzadeh, H.: J. Ind. Eng. Chem., 20, 2014, p. 4363. doi:10.1016/j.jiec.2014.01.045

[2] Ehsani, A., Mahjani, M. G., Moshrefi, R., Mostaanzadeh, H., Shabni-Shayeh, J.: RSC Advances, 4, 2014, p. 20031. doi:10.1039/c4ra01029a

[3] Bereket, G., Pinarbasi, A.: Corros. Eng. Sci. Techn., 39, 2004, p. 308. doi:10.1179/174327804X13136

[4] Khaled, K. F., Amin, M. A.: Corros. Sci., 51, 2009, p. 1964. doi:10.1016/j.corsci.2009.05.023

[5] Obot, I. B., Obi-Egbedi, N. O., Umoren, S. A.: Corros. Sci., 51, 2009, p. 276. doi:10.1016/j.corsci.2008.11.013

[6] Habibi, D., Nasrollahzadeh, M., Kamali, T. A.: Green Chem., 13, 2011, p. 3499. doi:10.1039/c1gc15245a

[7] Mahjani, M. G., Moshrefi, R., Ehsani, A., Jafarian, M.: Anti-Corros. Method. M, 58, 2011, p. 250. doi:10.1108/00035591111167721

[8] Ehsani, A., Mahjani, M. G., Jafarian, M.: Turk. J. Chem., 35, 2011, p. 1. doi:10.3906/kim-1103-50

[9] Ehsani, A., Mahjani, M. G., Babaei, F., Mostaanzadeh, H.: RSC Advances, 5, 2015, p. 30394. doi:10.1039/c5ra02297e

[10] Ehsani, A., Mahjani, M. G., Jafarian, M., Naeemy, A.: Electrochim. Acta, 71, 2012, p. 128. doi:10.1016/j.electacta.2012.03.107

[11] Ehsani, A., Vaziri-Rad, A., Babaei, F., Mohammad Shiri, H.: Electrochim. Acta, 159, 2015, p. 140. doi:10.1016/j.electacta.2015.01.204

[12] A. Ehsani, A., Babaei, F., Mostaanzadeh, H.: J. Braz. Chem. Soc., 26, 2015, p. 331. doi:10.5935/0103-5053.20140284

[13] Naghdi, S., Jaleh, B., Ehsani, A.: Bull. Chem. Soc. Jpn., 88, 2015, p. 722. doi:10.1246/bcsj. 20140402 
[14] Ehsani, A., Mahjani, M. G., Bordbar, M., Adeli, S.: J. Electroanal. Chem., 29, 2013, p. 710. doi:10.1016/j.jelechem.2013.01.008

[15] Ehsani, A.: Prog. Org. Coat., 78, 2015, p. 133. doi:10.1016/j.porgcoat.2014.09.015

[16] Ehsani, A., Mahjani, M. G., Nasseri, M., Jafarian, M.: Anti-Corros. Method. M, 61, 2014, p. 146. doi:10.1108/ACMM-07-2012-1193
[17] M. Curioni, M., Cottis, R., Di Natale, M., Thompson, G.: Electrochim. Acta, 56, 2011, p. 10270. doi:10.1016/j.electacta.2011.09.013

[18] Curioni, M., Balaskas, A., Thompson, G.: Corros. Sci., 77, 2013, p. 281. doi:10.1016/j.corsci.2013.08.014

[19] Lowe, A., Eren, H., Bailey, S.: Corros. Sci., 45, 2003, p. 941. doi:10.1016/S0010-938X(02)00179-8 